\title{
FOURIER TRANSFORM PERTURBATION SOLUTION OF ELLIPTIC EQUATIONS WITH SMALL NONLINEARITIES*
}

BY

\author{
S. C. CHIKWENDU
}

University of Nigeria, Nsukka

1. Introduction. Many linearized physical problems are described by Laplace's equation, for example in steady-state heat conduction, potential flow, theory of elasticity, and elsewhere. When the small nonlinear terms are retained the resulting elliptic equations are weakly nonlinear. Multiple-scale perturbation methods have been used [1] to solve certain weakly nonlinear elliptic equations in two independent variables. In that paper, a method which Chikwendu and Kevorkian [2] had found useful for nonlinear hyperbolic equations was extended to nonlinear elliptic equations, complex characteristics being used instead of real characteristics. However, only a very limited class of boundary-value problems (on a semi-infinite strip) was considered. Also, Eckhaus [3] has shown that the series used in [2] is uniformly asymptotically valid for space-periodic initial conditions (or boundary conditions in the elliptic case).

Recently, Chikwendu [4] has developed a Fourier transform perturbation method for nonlinear wave equations on the infinite line. In this note we wish to show that the Fourier transform perturbation method can be applied to weakly nonlinear elliptic equations on the upper half-plane, and that the boundary data need not be periodic. This should make the method more useful since it is well known that many boundary-value problems for Laplace's equation can be solved by conformal mapping to the upper half-plane.

Thus we consider the equation

$$
u_{y y}+u_{x x}+\varepsilon h\left(u, u_{x}, u_{y}, u_{x x}, \ldots\right)=0, \varepsilon \ll 1,-\infty<x<\infty, y>0,
$$

with appropriate Dirichlet $[u(x, 0 ; \varepsilon)=a(x)]$, Neumann or mixed boundary conditions specified on $y=0,-\infty<x<\infty$. We assume that $u(x, y ; \varepsilon)$ is bounded and Fouriertransformable with respect to $x$. It is also assumed that the nonlinearity $h$ has a Taylor series in its arguments and can be Fourier-transformed, for example in the form of convolutions.

The Fourier transform perturbation method involves Fourier-transforming Eq. (1.1) with respect to $x$ and then seeking a solution of the resulting ordinary differential equation for the Fourier transform of $u$ in the form of a uniformly valid asymptotic expansion in powers of $\varepsilon$. Multiple scales are used and "secular" terms are eliminated, as is done in nonlinear oscillations, leading to equations for the "slow" variation of the solution. These solutions are then Fourier-inverted back to physical space.

\footnotetext{
* Received October 15, 1981.
} 
2. Fourier transform perturbation method. We introduce the slow variable

$$
Y=\varepsilon y,
$$

and before Fourier-transforming we first seek a solution of Eq. (1.1) in the form of a uniformly valid asymptotic expansion in powers of $\varepsilon$,

$$
u(x, y ; \varepsilon)=\sum_{n=0}^{N} \varepsilon^{n} u_{n}(x, y, Y)+O\left(\varepsilon^{N+1}\right) .
$$

When this expansion (2.2) is used in Eq. (1.1), with $u_{y}$ now becoming $u_{y}+\varepsilon u_{Y}$ and the coefficients of $\varepsilon^{n}$ equated to zero for $n=0,1,2, \ldots$, a system of equations is obtained for $u_{0}, u_{1}, u_{2}, \ldots$ The first two equations are

$$
\begin{aligned}
& u_{0_{y y}}+u_{0_{x x}}=0, \\
& u_{1_{y y}}+u_{2_{x x}}=-2 u_{0_{y y}}-h\left(u_{0}, u_{0_{x}}, u_{0_{y}}, \ldots\right) .
\end{aligned}
$$

The dominant approximation $u_{0}$ is governed by Laplace's equation (2.3a) which has the general solution

$$
u_{0}(x, y, Y)=f(x-i y, Y)+g(x+i y, Y)
$$

where

$$
\alpha=x-i y, \quad \beta=x+i y
$$

are the complex characteristics. In the case of Dirichlet boundary conditions $a(x)$, the solution of Laplace's equation on the upper half-plane is given by Poisson's integral

$$
u_{0}(x, y, 0)=(1 / \pi) \int_{-\infty}^{\infty} \frac{y a(t) d t}{(x-t)^{2}+y^{2}}, \quad y>0,
$$

which can be written in the form of Eq. (2.4) as

$$
\begin{aligned}
u_{0}(x, y, 0) & =\frac{1}{2 \pi i} \int_{-\infty}^{\infty} \frac{a(t) d t}{\alpha-t}-\frac{1}{2 \pi i} \int_{-\infty}^{\infty} \frac{a(t) d t}{\beta-t} \\
& =f(\alpha, 0)+g(\beta, 0) .
\end{aligned}
$$

We note that $f$ and $g$ are complex conjugates and so $u_{0}(x, y, 0)=2 \operatorname{Re} f(\alpha, 0)$.

Fourier transformation. Using the Fourier transform definition

$$
U(\lambda, y, \varepsilon)=\int_{-\infty}^{\infty} e^{i \lambda x} u(x, y, \varepsilon) d x
$$

and its inverse

$$
u(x, y, \varepsilon)=\frac{1}{2 \pi} \int_{-\infty}^{\infty} e^{-i \lambda x} U(\lambda, y, \varepsilon) d \lambda,
$$

the expansion (2.2) can be Fourier-transformed, leading to the new expansion

$$
U(\lambda, y, Y, \varepsilon)=\sum_{n=0}^{N} \varepsilon^{n} U_{n}(\lambda, y, Y)+O\left(\varepsilon^{N+1}\right),
$$

where the Fourier transform of a (lower-case letter) function is denoted by the corresponding capitalized variable. 
The system of equations (2.3) thus transforms to

$$
\begin{aligned}
& U_{0_{y y}}-\lambda^{2} U_{0}=0, \\
& U_{1_{y y}}-\lambda^{2} U_{1}=-2 U_{0_{y y}}-H\left(U_{0}, U_{0_{y}},-i \lambda U_{0}, \ldots\right),
\end{aligned}
$$

and the solution of Eq. (2.10a) is

$$
U_{0}(\lambda, y, Y)=A(\lambda, Y) \exp (-|\lambda| y), y>0,
$$

where $A(\lambda, 0)$ is the Fourier transform of the boundary data on $y=0$. In this note it is convenient to write this solution (2.11) in the form of the Fourier transform of Eq. (2.4), as

$$
U_{0}(\lambda, y, Y)=F(\lambda, Y) e^{-\lambda y}+G(\lambda, Y) e^{\lambda y},
$$

where for boundedness we must have

$$
\begin{aligned}
F(\lambda, Y) & =A(\lambda, Y), & & \operatorname{Re} \lambda>0 \\
& =0, & & \operatorname{Re} \lambda<0, \\
G(\lambda, Y) & =0, & & \operatorname{Re} \lambda>0 \\
& =A(\lambda, Y), & & \operatorname{Re} \lambda<0 .
\end{aligned}
$$

Since $y$ appears in the solution (2.4) only in the form $i y$, we can define

$$
s=i y,
$$

and the leading approximation in Fourier space (2.12) can now be written as

$$
U_{0}(\lambda, s, Y)=F(\lambda, Y) e^{i \lambda s}+G(\lambda, Y) e^{-i \lambda s} \text {. }
$$

This is an important step because, instead of exponential decay with $y, U_{0}$ is now oscillatory in $s$, and so the methods of nonlinear oscillations can be applied to elliptic equations as has been done for wave equations [4]. We thus proceed to find the $Y$-dependence of $F$ and $G$ by eliminating "secular" terms from Eq. (2.10b) in the usual manner for ordinary differential equations (Cole [5]).

Using (2.14) and (2.15), the $O(\varepsilon)$ equation (2.10b) can be written as

$$
U_{1, s}+\lambda^{2} U_{1}=-2 \lambda F_{Y} e^{i \lambda s}+2 \lambda G_{Y} e^{-i \lambda s}+H\left(U_{0}, i U_{0_{s}},-i \lambda U_{0}, \ldots\right) \text {. }
$$

In order that the asymptotic expansion (2.9) remain consistent and uniform (avoiding indefinite growth with $Y$ when $\lambda$ and $s$ are held fixed), the inhomogeneous term on the right-hand side of (2.16) must be orthogonal to all the solutions of the corresponding homogeneous problem. If we assume that $u$ is symmetric in $y$ (i.e. that its behavior in the lower half-plane is the same as in the upper half-plane), then we can multiply (2.16) by $\exp (i \lambda s)$ or $\exp (-i \lambda s)$, integrate with respect to $s$ from $s=-M$ to $s=M$, divide by $2 M$ and take the limit as $M \rightarrow \infty$. After integration by parts the left-hand side is seen to go to zero. The resulting slowly varying equations are

$$
\begin{gathered}
2 \lambda F_{Y}(\lambda, Y)-\lim _{M \rightarrow \infty} \frac{1}{2 M} \int_{-M}^{M} e^{-i \lambda s} H\left(U_{0}, i U_{0_{s}}, \ldots\right) d s=0, \\
2 \lambda G_{Y}(\lambda, Y)+\lim _{M \rightarrow \infty} \frac{1}{2 M} \int_{-M}^{M} e^{i \lambda s} H\left(U_{0}, i U_{0_{s}}, \ldots\right) d s=0 .
\end{gathered}
$$

Since $U_{0}$ is periodic in $s$ with frequency $\lambda$ (from Eq. (2.15)), it follows that $H$ will also be 
periodic in $s$ and so can be written as a sum of terms with frequencies $\lambda, 2 \lambda, 3 \lambda, \ldots$ Therefore in the limit as $M \rightarrow \infty$ the averaged integrals in Eqs. (2.17) will only pick up the terms with frequency $\lambda$ since all the others will be orthogonal to $e^{-i \lambda s}$ (or $e^{i \lambda s}$ ). These averaged integrals will thus be independent of $s$.

The equations (2.17) are therefore a pair of coupled nonlinear integrodifferential equations for $F$ and $G$. If these equations are solved, $F$ and $G$ can then be Fourier-inverted to give the lowest-order solution $u_{0}$, and the process can be continued to determine the higher-order approximations.

Convolution nonlinearities. For hyperbolic equations [4] interesting results were obtained when the nonlinearity was of convolution form, but for elliptic equations the results are not as interesting. Consider, for example, the equation

$$
u_{y y}+u_{x x}+\varepsilon \int_{-\infty}^{\infty} \int_{-\infty}^{\infty} u(x-\xi, y) u(\xi-\eta, y) u(\eta, y) d \xi d \eta=0
$$

Fourier-transformation of this equation immediately gives

$$
U_{y y}-\lambda^{2} U+\varepsilon U^{3}=0
$$

and in this case Eq. (2.10b) becomes

$$
U_{1_{y y}}-\lambda^{2} U_{1}=-2 U_{0 y Y}-U_{0}^{3} .
$$

The nonlinearity will not contribute secular terms and Eqs. (2.17) can be used to show that $U_{0_{Y}} \equiv 0$. This is perhaps most immediately seen if Eq. (2.11) is used in (2.20), giving

$$
U_{1_{y y}}-\lambda^{2} U_{1}=2|\lambda| A_{Y}(\lambda, Y) \exp (-|\lambda| y)-A^{3}(\lambda, Y) \exp (-3|\lambda| y) .
$$

Thus the nonlinear inhomogeneous term does not contain terms which are in the form of the solutions of the homogeneous problem, and so $A$ will be independent of $Y$. In this problem $U$ posseses a straight-forward, regular asymptotic expansion.

3. Illustrative example. Consider the boundary-value problem

$$
\begin{aligned}
u_{y y}+u_{x x}-\varepsilon\left(u_{y}-u u_{y}\right) & =0, y>0, \\
u(x, 0) & =2\left(1+e^{x}\right)^{-1} .
\end{aligned}
$$

For this nonlinear problem, Eq. (2.17a) becomes

$$
\begin{aligned}
2 \lambda F_{Y}(\lambda, Y)-\lambda F(\lambda, Y) & -\lim _{M \rightarrow \infty} \frac{1}{(2 \pi) 2 M} \int_{-M}^{M} e^{-i \lambda s} d s \int_{-\infty}^{\infty}\left[F(\lambda-\eta, Y) e^{i(\lambda-\eta) s}\right. \\
& \left.+G(\lambda-\eta, Y) e^{-i(\lambda-\eta) s}\right] \eta\left[G(\eta, Y) e^{-i \eta s}-F(\eta, Y) e^{i \eta s}\right] d \eta=0,
\end{aligned}
$$

that is,

$$
\begin{aligned}
& 2 \lambda F_{Y}(\lambda, Y)-\lambda F(\lambda, Y)+(1 / 2 \pi) \int_{-\infty}^{\infty} F(\lambda-\eta, Y) \eta F(\eta, Y) d \eta \\
&-\lim _{M \rightarrow \infty} \frac{1}{(2 \pi) 4 M}\left[\int_{-\infty}^{\infty} F(\lambda-\eta, Y) \eta G(\eta, Y) d \eta \int_{-2 M}^{2 M} e^{-i \eta t} d t-\int_{-\infty}^{\infty} G(\lambda-\eta, Y) \eta F(\eta, Y) d \eta\right. \\
&\left.\int_{-2 M}^{2 M} e^{-i(\lambda-\eta) t} d t+\int_{-\infty}^{\infty} G(\lambda-\eta, Y) \eta G(\eta, Y) d \eta \int_{-2 M}^{2 M} e^{-i \lambda t} d t\right]=0,
\end{aligned}
$$

where $t=2 s$. 
Under the limit sign in Eq. (3.3) we can interchange the limits of the $t$ and $\eta$ integrals. Thus instead of integrating on the strip $-\infty<\eta<\infty,-2 M<t<2 M$, we can integrate on the strip $-2 M<\eta<2 M,-\infty<t<\infty$ before letting $M \rightarrow \infty$. In both cases the entire $\eta, t$ plane is covered. With this interchange, Eq. (3.3) can be written as

$$
\begin{aligned}
2 \lambda F_{Y}(\lambda, Y)-\lambda F(\lambda, Y)+(1 / 2 \pi) \int_{-\infty}^{\infty} F(\lambda-\eta, Y) \eta F(\eta, Y) d \eta \\
-\lim _{M \rightarrow \infty} \frac{1}{(2 \pi) 4 M}\left[\int_{-2 M}^{2 M} F(\lambda-\eta, Y) \eta G(\eta, Y) d \eta \int_{-\infty}^{\infty} e^{-i \eta t} d t-\int_{-2 M}^{2 M} G(\lambda-\eta, Y) \eta F(\eta, Y) d \eta\right. \\
\\
\left.\cdot \int_{-\infty}^{\infty} e^{-i(\lambda-\eta) t} d t+\int_{-2 M}^{2 M} G(\lambda-\eta, Y) \eta G(\eta, Y) d \eta \int_{-\infty}^{\infty} e^{-i \lambda t} d t\right]=0 .
\end{aligned}
$$

Since $\int_{-\infty}^{\infty} e^{-i \eta t} d t=2 \pi \delta(\eta)$, where $\delta$ is the Dirac delta function, Eq. (3.4) becomes

$$
\begin{aligned}
2 \lambda F_{Y}(\lambda, Y)-\lambda F(\lambda, Y)+(1 / 2 \pi) & \int_{-\infty}^{\infty} F(\lambda-\eta, Y) \eta F(\eta, Y) d \eta \\
+ & \lim _{M \rightarrow \infty} \frac{1}{4 M}\left[\lambda F(\lambda, Y) \int_{-2 M}^{2 M} G(\lambda-\eta, Y) \delta(\lambda-\eta) d \eta\right. \\
& \left.-\int_{-2 M}^{2 M} G(-\eta, Y) \eta G(\eta, Y) d \eta\right]=0,
\end{aligned}
$$

or

$$
\begin{aligned}
2 \lambda F_{Y}(\lambda, Y)-\lambda F(\lambda, Y) & +(1 / 2 \pi) \int_{-\infty}^{\infty} F(\lambda-\eta, Y) \eta F(\eta, Y) d \eta \\
& +\lambda F(\lambda, Y)\langle G(-\xi, Y) \delta(\xi)\rangle-\langle G(\lambda-\xi, Y) \xi G(\xi, Y) \delta(\lambda)\rangle=0,
\end{aligned}
$$

where,

$$
\langle\cdots\rangle=\lim _{M \rightarrow \infty} \frac{1}{2 M} \int_{-M}^{M} \cdots d \xi .
$$

If Eq. (3.6) is Fourier-inverted back to the physical plane the resulting equation is

$$
2 f_{\alpha Y}-f_{\alpha}+f f_{\alpha}+f_{\alpha}\langle g(\beta, Y)\rangle=\left\langle g g_{\beta}\right\rangle .
$$

But

$$
\left\langle g g_{\beta}\right\rangle=\lim _{M \rightarrow \infty} \frac{1}{2 M} \int_{-M}^{M} g g_{\beta} d \beta=\lim _{M \rightarrow \infty} \frac{1}{4 M}\left[g^{2}(M, Y)-g^{2}(-M, Y)\right]=0,
$$

since $g$ is bounded.

Thus after integration with respect to $\alpha$, Eq. (3.8) becomes

$$
2 f_{Y}-f[1-\langle g\rangle]+f^{2} / 2=0 .
$$

Similarly, from (2.17b), the corresponding equation for $g$ is

$$
2 g_{Y}-g[1-\langle f\rangle]+g^{2} / 2=0 .
$$


Eq. (3.10a) is a Bernoulli ordinary differential equation and can be linearized through the substitution $v=1 / f$. Alternatively, since $\langle g\rangle$ is a function of $Y$ only, the solution can be written as

$$
f(\alpha, Y)=4 \phi^{\prime}(Y) f(\alpha, 0)[\phi(Y) f(\alpha, 0)+1]^{-1},
$$

where

$$
4 \phi^{\prime}(Y)=\exp \left\{(1 / 2) \int_{0}^{Y}\left[1-\langle g\rangle\left(Y^{\prime}\right)\right] d Y^{\prime}\right\} ; \phi(0)=0 .
$$

Since $f$ and $g$ are complex conjugates and here satisfy similar equations $(3.10 \mathrm{a}, \mathrm{b})$, it follows that for this example, $\langle g\rangle=\langle f\rangle$, and

$$
g(\beta, Y)=4 \phi^{\prime}(Y) g(\beta, 0)[1+\phi(Y) g(\beta, 0)]^{-1} .
$$

It can be shown that for the boundary condition (3.1b), the solution of Laplace's equation on the upper half-plane (2.7) is obtained from

$$
f(\alpha, 0)=\left(1+e^{\alpha}\right)^{-1} ; g(\beta, 0)=\left(1+e^{\beta}\right)^{-1},
$$

and so

$$
f(\alpha, Y)=4 \phi^{\prime}(Y)\left[\phi(Y)+1+e^{\alpha}\right]^{-1},
$$

and using the definition (3.7), we have

$$
\langle f\rangle=\langle g\rangle=2 \phi^{\prime}(Y) /[\phi(Y)+1] .
$$

The differential equation (3.12) for $\phi$ can thus be written as

$$
\log \left(4 \phi^{\prime}(Y)\right)=Y / 2-\log [\phi(Y)+1]
$$

or

$$
4 \phi^{\prime}(Y)[\phi(Y)+1]=e^{Y / 2} ; \phi(0)=0 .
$$

The solution of (3.18) for $\phi$ is

$$
\phi(Y)=\exp (Y / 4)-1
$$

and using this in (3.15) gives

$$
f(\alpha, Y)=\exp (Y / 4) /[\exp (Y / 4)+\exp (x-i y)] .
$$

Finally, the leading approximation to the solution of the nonlinear boundary-value problem (3.1) is

$$
u_{0}(x, y, Y)=2 \operatorname{Re} f(\alpha, Y)=\frac{2 e^{Y / 4}\left(e^{Y / 4}+e^{x} \cos y\right)}{\left[\left(e^{Y / 4}+e^{x} \cos y\right)^{2}+\left(e^{x} \sin y\right)^{2}\right]} .
$$

An interesting approach of the dominant solution to a constant at large $Y$ has thus been determined.

\section{REFERENCES}

[1] S. C. Chikwendu, Asymptotic solutions of some weakly nonlinear elliptic equations, SIAM J. Appl. Math. 31, 286-303 (1976) 
[2] S. C. Chikwendu and J. Kevorkian, A perturbation method for hyperbolic equations with small nonlinearities, SIAM J. Appl. Math. 22, 235-258 (1972)

[3] W. Eckhaus, New approach to the asymptotic theory of nonlinear oscillations and wave propagation, J. Math. Anal. \& Applics. 49, 575-611 (1975)

[4] S. C. Chikwendu, Nonlinear wave propagation solution by Fourier transform perturbation, Int. J. Nonlinear Mech. 16, 117-128 (1981)

[5] J. D. Cole, Perturbation methods in applied mathematics, Blaisdell, Waltham, Mass., 1968 